\title{
CONSUMO DE PLÁTANO EN ECUADOR: HÁBITOS DE COMPRA Y DISPONIBILIDAD A PAGAR DE LOS CONSUMIDORES
}

\author{
Wilmer S. Sepúlveda* \\ Irinuska Ureta* \\ Gema A. Hernández*** \\ Gissell K. Solorzano*****
}

\begin{abstract}
RESUMEN: El plátano es uno de los productos tradicionales del Ecuador. El objetivo del presente estudio fue analizar hábitos de compra del plátano en Ecuador y la disponibilidad a pagar de los consumidores por atributos ligados con la producción. La información usada en el estudio, proviene de 400 encuestas realizadas a consumidores. Asimismo, se desarrolló un experimento de elección para evaluar la DAP de los consumidores por diversos atributos del producto. Las principales elaboraciones culinarias son los patacones y bolones. En la diferenciación del producto, la producción ética, el uso de un estándar de calidad, la producción orgánica y el origen, pueden desempeñar un rol importante.
\end{abstract}

PALABRAS CLAVE: Estudio de mercado; Experimento de elección; Calidad.

\section{PLANTAIN CONSUMPTION IN ECUADOR: PURCHASE HABITS AND CONSUMERS' WILLINGNESS TO PAY FOR PRODUCTION ATTRIBUTES}

\begin{abstract}
The plantain is one of the most traditional products in Ecuador. The aim of the present study was to analyze plantain purchase habits by consumers in Ecuador and the WTP of consumers toward several attributes in the plantain production. The information used in the study, comes from 400 consumer' surveys. The surveys included a choice experiment to evaluate consumers' WTP for different product

Doctor en Avances en Ciencias Agrarias y del Medio Natural, Integrante del Grupo de Investigación IPER, Profesor de la Facultad de Ciencias Económicas, Administrativas y Contables, Universidad Libre, Campus Majavita, El Socorro, Colombia

** Máster en Ciencias Sociales, Profesora de Tiempo Completo de la Escuela de Economía, Facultad de Ciencias Administrativas y Económicas, Universidad Técnica de Manabí, Portoviejo, Ecuador. Autor de correspondencia, e-mail:miureta@utm.edu.ec

*** Economista, Egresada de la Escuela de Economía, Facultad de Ciencias Administrativas y Económicas, Universidad Técnica de Manabí, Portoviejo, Ecuador.

**** Economista, Egresada de la Escuela de Economía, Facultad de Ciencias Administrativas y Económicas, Universidad Técnica de Manabí, Portoviejo, Ecuador
\end{abstract}


attributes. Patacones and bolones are the principal meals in the Ecuadorians' home. For the differentiation of plantain production toward consumers, ethical production, quality standard label, organic production and origin, can play and important role.

KEY WORDS: Market research; Choice experiment analysis; Quality.

\section{INTRODUCCIÓN}

El banano y el plátano (Musa spp.) son de los productos más emblemáticos del trópico (Noceda et al., 2012; Lynam, 2000). Aunque el término banano abarca todos los miembros del género Musa, en sentido estricto, el plátano hace referencia a un grupo específico definido dentro de este género (Jekayinfa, Ola, Afolayan \& Ogunwale, 2012). A diferencia del banano, el plátano es una fruta que debido a su alto contenido de almidón debe ser cocida antes de ser consumida, siendo así conocida como plátano para cocinar o bananas de cocción (Giraldo Toro et al., 2015; Tchango, Bikoï, Achard, Escalant \& Ngalani, 1999). En este sentido, la palabra "banano" se usa para referirse a las especies Musa que son más dulces y se consumen crudas mientras que la palabra "plátano", se usa para denotar las especies Musa que son cocidas antes de ser consumidas (Cauthen \& Jones, 2013). El plátano se caracteriza por el color naranja-amarillo de la pulpa una vez ha alcanzado su maduración. Los frutos son delgados y de forma angular en la punta (Jekayinfa, Ola, Afolayan \& Ogunwale, 2012). Generalmente se cultiva en asocio con plantaciones de café y cacao. Es un alimento básico para millones de personas a nivel mundial (Tchango, Bikö̈, Achard, Escalant \& Ngalani, 1999). Junto con las raíces y los tubérculos, son fuentes importantes de energía para la población del trópico (Lynam, 2000). El plátano es ampliamente consumido en diversas preparaciones (Dury, Bricas, Tchango-Tchango, Temple \& Bikoi, 2002).

A nivel global, el $72,7 \%$ de la producción de plátano se concentra en África, seguido por América (22,9\%) y Asia (4,3\%) (FAOSTAT). En el continente americano, los mayores productores son Colombia (38,1\%), Perú (21,6\%), República Dominicana $(6,9 \%)$ y Ecuador (6,4\%) (Cálculos propios a partir de FAOSTAT). En cuanto al comercio, en el año 2014 se exportó plátano a nivel global por un valor de 470,2 millones de dólares. Con 97,3 millones de dólares, Ecuador ocupa el primer 
lugar en las exportaciones mundiales (TradeMap). Por tanto, en Ecuador, el plátano además de ser un producto tradicional, su producción genera importantes divisas para el país. De acuerdo con el Instituto Nacional de Estadística y Censos (INEC), en Ecuador hay plantadas un estimado de 141.441 hectáreas de plátano. Con 50.376 hectáreas, la provincia de Manabí ubicada al sur-occidente del país es la mayor productora concentrando el 35,6\% del área total. A nivel nacional, el 71,6\% de los cultivos de plátano son establecidos como monocultivo lo que indica cierto grado de especialización de la producción (cálculos propios a partir de INEC). El plátano se comercializa principalmente en el mercado regional. Sin embargo, la prevalencia de bajos precios en épocas de sobreproducción (invierno) en contraposición con los costos y la alta intermediación en la cadena de proveeduría sumado a su carácter de commodity, hace presión en la generación de productos diferenciados y en la necesidad de incursionar directamente en los mercados nacionales por parte de las asociaciones de productores (Sepúlveda, 2015). Según los agricultores de la región, el mayor problema que se presenta en la producción de plátano es la comercialización (Mendoza, 2015). Dicha problemática va de la mano con la generación de cadenas enfocadas hacia el mercado, en donde el conocimiento del consumidor es de vital importancia para el desarrollo de acciones generadoras de valor (Sepúlveda, Maza \& Pardos, 2011). En este sentido, conocer los hábitos de compra de los consumidores y los atributos que podrían generar más diferenciación ante los mismos, podría apoyar en la toma de decisiones por parte de los actores de la cadena de plátano del Ecuador.

En línea con lo anterior, el objetivo de la presente investigación llevada a cabo en Ecuador, fue analizar los hábitos de compra/consumo de plátano por parte de los consumidores y su disponibilidad a pagar por diferentes atributos ligados con la producción que podrían ejercer un efecto diferenciador. A pesar de ser un producto común en la dieta tropical, la literatura a nivel mundial sobre estudios de los consumidores respecto al consumo de plátano parece ser limitada (Dury, Bricas, Tchango-Tchango, Temple \& Bikoi, 2002; Honfo, Tenkouano \& Coulibaly, 2011; Cortes \& Gayol, 2012). Dury, Bricas, Tchango-Tchango, Temple y Bikoi (2002), desarrollaron un estudio en Camerún con el fin de identificar los hábitos de consumo y aspectos de calidad relativos al plátano. Asimismo, en un estudio llevado a cabo en Nigeria y Camerún, Honfo, Tenkouano y Coulibaly (2011), reportaban 
altos niveles de consumo de plátano por parte de la población. Por su parte, Cortes y Gayol (2012) en un estudio llevado a cabo en Puerto Rico, analizaron los diferentes atributos del plátano que son más demandados por los consumidores. El desarrollo del presente estudio es relevante desde el punto de vista de contribución a la literatura y de la generación de información para apoyar la toma de decisiones por parte de los productores.

\section{METODOLOGÍA}

La información empleada en el presente estudio, proviene del desarrollo de 400 encuestas dirigidas a consumidores responsables de la compra de alimentos en sus hogares residentes en Guayaquil (212), Quito (149) y el área metropolitana de Manta-Portoviejo (39), tres de los mayores centros urbanos de Ecuador. La distribución del número de observaciones de la muestra por ciudades, obedeció al desarrollo de un muestreo estratificado mediante afijación proporcional por tamaño de la población. El levantamiento de la información tuvo lugar durante el mes de abril del año 2015. Asignando a p y $q$ valores intermedios $(p=q=0,5)$ y para un intervalo de confianza en los resultados del $95,5 \%$, el máximo error que se pudo cometer fue del 5\%. En la tabla 1 se presenta información sobre las características sociodemográficas de la muestra.

Tabla 1. Características sociodemográficas de la muestra

(Continua)

\begin{tabular}{cc}
\hline Características & Porcentaje \\
\hline Género & \\
\hline Hombre & 54,5 \\
Mujer & 45,5 \\
\hline Nivel de estudio & \\
\hline Básico/primaria & 9,0 \\
Bachillerato/técnico & 56,0 \\
Tecnólogo & 4,8 \\
\hline
\end{tabular}


(Conclusão)

\begin{tabular}{cc}
\hline Características & Porcentaje \\
\hline Profesional & 30,3 \\
\hline Rango de edad & 7,5 \\
\hline Igual o menor a 20 años & 40,5 \\
Entre 21 y 30 años & 27,0 \\
Entre 31 y 45 años & 19,0 \\
Entre 46 y 60 años & 6,0 \\
Más de 60 años & \\
\hline Nivel de ingresos & 56,4 \\
Menos de USD \$750/mes & 30,1 \\
\hline Entre USD \$751/mes y USD \$1.500/mes & 8,1 \\
Entre USD \$1.501/mes y USD \$2.500/mes & 5,5 \\
Más de USD \$2.500/mes & \\
\hline
\end{tabular}

La muestra estuvo conformada en un $54,5 \%$ por hombres y $45,5 \%$ por mujeres. Existe un alto porcentaje de encuestados con niveles de estudio de bachillerato/técnico (56,0\%), seguido por el nivel profesional (30,3\%), básico/ primaria $(9,0 \%)$ y tecnológico $(4,8 \%)$. En cuanto a la edad, el 7,5\% poseían menos de 20 años, el 40,5\% entre 21 y 30 años, el 27,0\% entre 31 y 45 años, el 19,0\% entre 46 y 60 años y el 6,0\% más de 60 años. Con relación al nivel de renta, el mayor porcentaje estuvo representado por personas con niveles de ingresos familiares inferiores a USD $\$ 750 /$ mes $(56,4 \%)$, seguido por los niveles de ingresos de USD $\$ 751 /$ mes a USD $\$ 1.500 /$ mes $(30,1 \%)$.

La encuesta empleada para la recolección de la información, incluyó diversos bloques de preguntas. En particular uno de ellos estuvo dirigido a capturar los hábitos de compra/consumo del plátano (verde) como fruta fresca. Asimismo, la encuesta incluyó el desarrollo de un experimento de elección. El análisis estadístico de la información fue de tipo univariante, bivariante y multivariante empleando los software SPSS y SMRT. Los análisis univariantes se centraron en el cálculo de 
porcentajes y frecuencias. El test de Chi-cuadrado fue la técnica empleada en los análisis bivariantes.

El experimento de elección como método estadístico multivariado, fue empleado con el fin de calcular las utilidades generadas ante los consumidores por la producción ética, un estándar de calidad voluntario, la producción orgánica, el origen y el precio en el plátano verde. El estándar de calidad empleado en el análisis fue uno no existente en el mercado nacional (estándar hipotético), pero similar al plátano clasificado como tipo A de exportación. Dicho estándar fue denominado Premium Quality (tipo exportación). La producción ética hace alusión al uso o no del sello de calidad de comercio justo "pequeños productores", mientras que como sello de producción orgánica se empleó el del Departamento de Agricultura de los Estados Unidos de América (USDA). Además de Manabí, como orígenes se incluyeron Santo Domingo y Guayas, debido a su posición importante en la producción nacional de plátano. Estos cuatro atributos, producción ética, producción orgánica, estándar de calidad voluntario (Premium Quality) y el origen, son considerados atributos de calidad superior que podrían suponer un efecto diferenciador en el mercado. Según Verbeke, Demey, Bosmans y Viaene (2005), los atributos de calidad superior son iniciativas de adopción voluntaria empleadas por los productores para resaltar la calidad ante los consumidores, logrando así un efecto diferenciador de la producción. Contrario al plátano, en el sector del banano existen algunos estudios reportados sobre el uso de atributos de calidad superior por parte de los agricultores (Trauger, 2015; Moberg, 2005; Fort \& Ruben, 2008). En la tabla 2 se presentan los atributos y niveles de los mismos tenidos en cuenta en el estudio, así como las respectivas codificaciones incluidas en el modelo. El precio base de USD $\$ 0,60$ por $\mathrm{Kg}$ de plátano verde, corresponde a precios reales de la fruta en Ecuador. 
Tabla 2. Atributos y niveles incluidos en el estudio

\begin{tabular}{ll}
\hline Atributo & Niveles \\
\hline Producción ética (pro.etica) & $-1=$ Sin sello de pequeños productores \\
1 & $=$ Con sello de pequeños productores \\
& $-1=$ Sin sello de calidad premium \\
Calidad (calidad) & $=$ Con sello de calidad premium \\
Sistema de producción (pro.organica) & $-1=$ Sin sello de producción orgánica \\
& $1=$ Con sello de producción orgánica \\
& $1=$ No específico \\
& $2=$ Manabí \\
3 & $=$ Santo Domingo \\
& $4=$ Guayas \\
Lugar de origen (origen) & $0,60=$ USD $\$ 0,60$ \\
& $0,78=$ USD $\$ 0,78$ \\
& $0,96=$ USD $\$ 0,96$ \\
& $1,14=$ USD $\$ 1,14$ \\
\hline
\end{tabular}

Los experimentos de elección se basan en la maximización de la utilidad por parte de las personas. Esta técnica parte del supuesto de que una persona elige, entre dos o más alternativas mutuamente excluyentes, aquella que le proporcione una utilidad superior con relación a las demás disponibles en su marco de elección. Desde el punto de vista práctico, el consumidor se enfrenta ante dos o más productos hipotéticos diferentes, conformados cada uno por la combinación de los niveles de atributos predefinidos, de tal forma que el individuo elegirá aquel producto que le genere mayores utilidades y vaya más en línea con sus preferencias.

Con los atributos predefinidos (ver tabla 2), se diseñaron tarjetas de simulación. Estas incluían cada una dos opciones de productos hipotéticos A y B para que el consumidor eligiese uno de ellos existiendo la posibilidad de no elegir ningún producto. El experimento incluyó 16 tarjetas de simulación, las cuales fueron calculadas mediante un diseño ortogonal empleando el software SPSS. A modo de ejemplo, en la figura 1 se presenta la tarjeta 3 incluida en el estudio. 


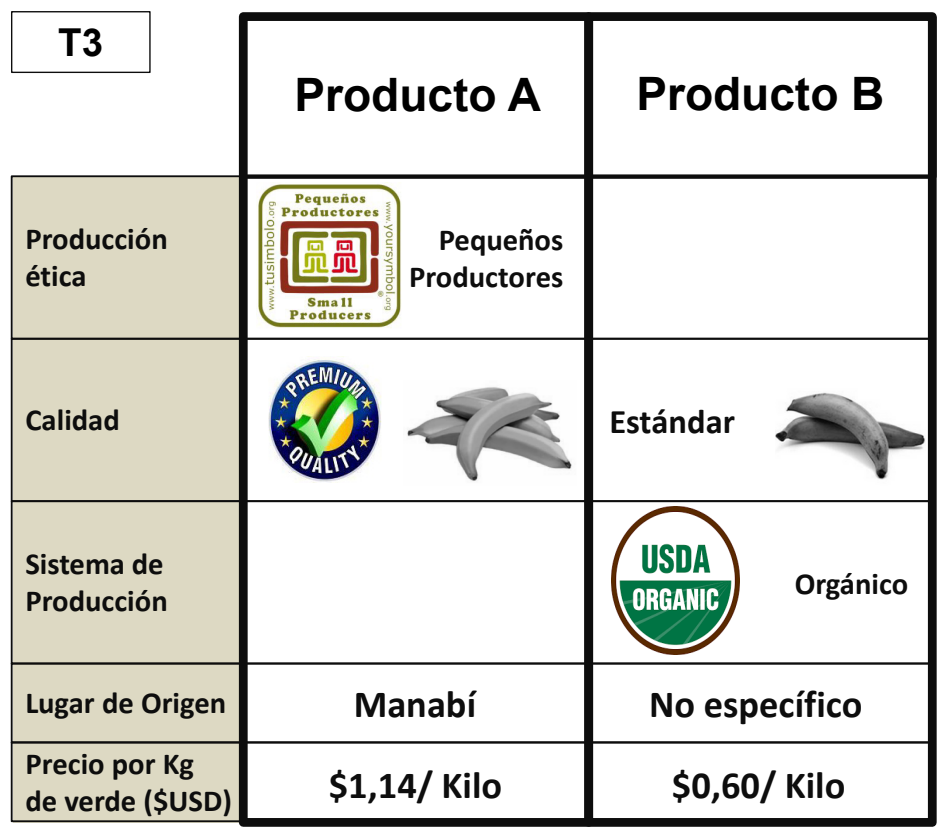

No Compraría ni el Producto A ni el Producto B

Figura 1. Ejemplo de tarjetas empleadas en el estudio: tarjeta 3

El cálculo de las utilidades se basó en la regresión logística condicional de McFaden (2001):

$\operatorname{Prob}_{i, J}(j)=\frac{e^{B / X_{i j}}}{\sum_{k \in J} e^{B / X_{i k}}}$

Teniendo en cuenta los atributos empleados en el experimento de elección, el modelo quedaría especificado de la siguiente manera:

$\operatorname{Prob}_{i, J}(j)=\frac{e^{V_{i j}}}{\sum_{k \in J} e^{V_{i k}}}$

$\operatorname{Prob}_{i}(A)=\frac{e^{V_{i A}}}{e^{V_{i A}}+e^{V_{i B}}} \quad$ y, $\quad \operatorname{Prob}_{i}(B)=\frac{e^{V_{i B}}}{e^{V_{i A}+e^{V_{i B}}}}$ 
Donde;

$V i_{(A, B)}=\alpha+\beta 1$. pro.etica $+\beta 2$. calidad $+\beta 3$. pro.organica $+\beta 4$. origen $+\beta 5$. precio

El valor de $\alpha$ captura el efecto dado por la opción "no compraría ni el producto A ni el producto B", es decir, captura el efecto generado por atributos no incluidos en el modelo. En este sentido, para $\alpha, 0=$ si seleccionó algún perfil (A ó B) y 1 = no seleccionó ninguno de los dos perfiles expuestos en cada una de las 16 tarjetas (Gracia, López \& Virué, 2011).

Para analizar el ajuste del modelo se empleó el índice RLH (root likelibood), donde un valor cercano a 1 indica un buen ajuste del modelo. Para la significancia de los parámetros estimados, se tuvo en cuenta el t-ratio de cada uno de los parámetros asociados a las variables incluidas en el modelo. Por su parte, la disposición a pagar por cada atributo diferente al precio, se obtuvo a través de la división de la derivada parcial de la función de utilidad respecto a cada atributo de interés, entre la derivada parcial de la función de utilidad con respecto al atributo precio (Gracia, López \& Virué, 2011).

\section{RESULTADOS Y DISCUSIÓN}

\subsection{HÁBITOS DE COMPRA/CONSUMO DE PLÁTANO (VERDE)}

Todos los consumidores encuestados poseen algún grado de responsabilidad en la compra de alimentos en sus hogares. El 46,8\% manifestaron ser siempre los responsables de la compra de alimentos, el 29,3\% casi siempre y el 24,0\% restante manifestaron ser de vez en cuando los responsables de la compra de alimentos en sus hogares.

De los tres productos analizados, yuca, camote y plátano, que podrían considerarse de cierta forma sustitutos entre sí, el camote es el que posee menores frecuencias de consumo, seguido por la yuca y el plátano (ver figura 2). Los resultados sugieren que este último es un producto altamente consumido en Ecuador; en el $77,3 \%$ de los hogares se consume al menos 3 veces por semana. Sin embargo, la frecuencia de consumo tiende a ser superior en las ciudades costeras como Manta, 
Guayaquil y Portoviejo e inferior en Quito (test de Chi cuadrado, $P=0,000$ ). De acuerdo a la Encuesta Nacional de Salud y Nutrición (ENSANUT, 2013), en Ecuador, el plátano es el quinto alimento que más aporta carbohidratos, después del arroz, el azúcar, el pan y la papa. Afonso (2014), en un estudio realizado en Ecuador para caracterizar la dieta alimentaria de la población rural de la provincia costera de Manabí, encontró que los cereales, azúcares y el plátano, eran las principales fuentes de aporte energético. En el mismo estudio (Afonso, 2014) se recalcaba sobre un consumo excesivo de carbohidratos por parte de la población rural, principalmente el arroz.

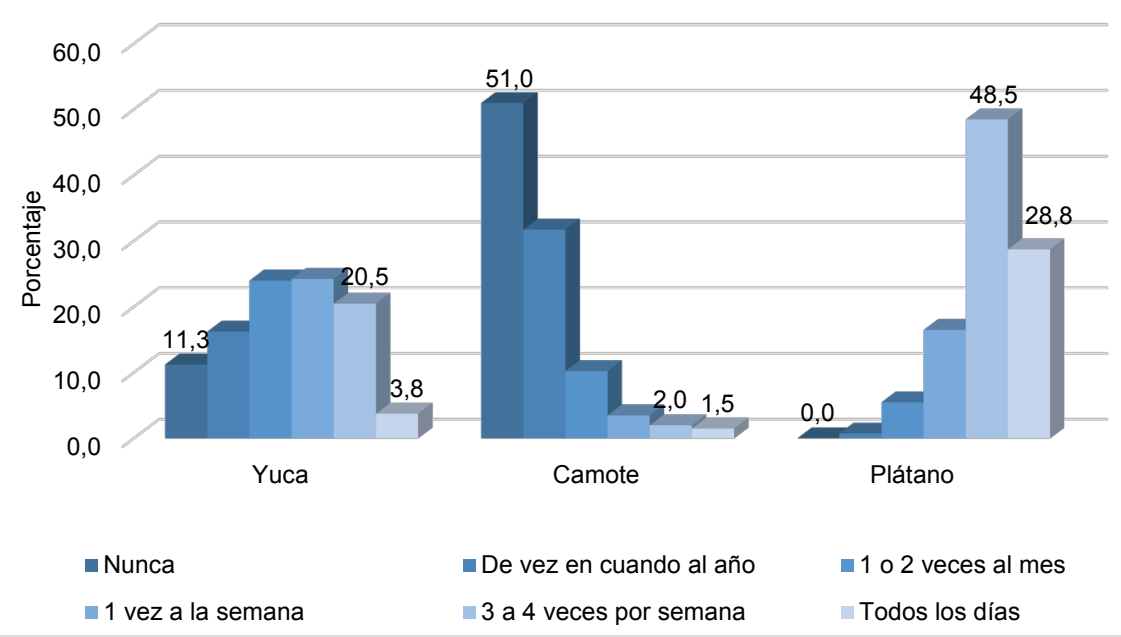

Figura 2. Frecuencia de consumo de plátano y sustitutos (en los hogares)

Se encontró que los principales canales comerciales empleados para la adquisición de plátano son las plazas de mercado (44,3\%) y las tiendas tradicionales y los micromercados (27,8\%). Los supermercados como lugar frecuente para la adquisición de plátano, son empleados por el 22,0\% de los consumidores. Canales como los hipermercados $(0,5 \%)$ y la compra directa al productor $(4,3 \%)$ son los menos recurrentes. Los resultados indican que existe relación de dependencia significativa entre el lugar frecuente empleado para la compra de plátano y la ciudad (test de Chi-cuadrado, $P=0,000$ ). Esto en el sentido de que las personas ubicadas en la ciudad de Quito (no costera) en comparación con las demás ciudades (costeras), 
tienden a adquirir en mayor medida plátano a través de los super/hipermercados (ver figura 3). En un estudio realizado en una ciudad de tamaño intermedio en Colombia, Gamboa, López, Prada, Franco y Landínez (2010) encontraron, que el principal canal comercial empleado por los consumidores para la adquisición de frutas y verduras son las plazas de mercado y las tiendas de barrio. Sin embargo, en contraposición a ello, Santo de Souza et al. (2008) en un estudio realizado en Brasil y Parrado y Montolla (2007) en un estudio llevado a cabo en Bogotá, reportaron que el principal canal comercial empleado para la adquisición de frutas y verduras son los súper/hipermercados. En el caso de Ecuador, los resultados sugieren que los canales de distribución tradicionales como plazas de mercado y tiendas de barrio aún juegan un importante papel en la adquisición de plátano y las demás futas y verduras por parte de los consumidores, principalmente en las ciudades costeras. Al igual que Benalcázar (2009), se encontró que en Quito existe una mayor preferencia por los supermercados.

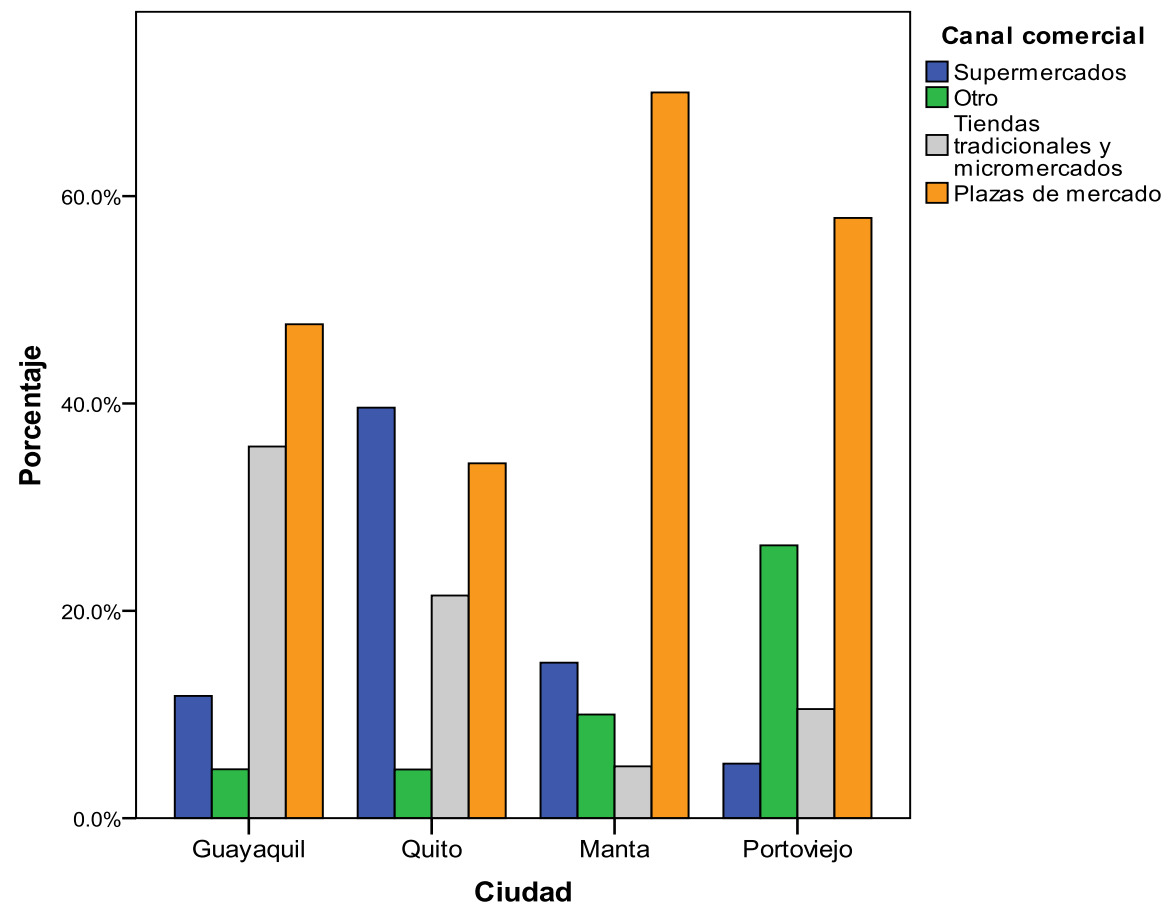

Figura 3. Lugar frecuentemente empleado para la compra de plátano por ciudad 
Otro aspecto a resaltar, es que las personas con mayores niveles de estudio (test de Chi-cuadrado, $P=0,000$ ) y mayores niveles de ingresos (test de Chi-cuadrado, $P=0,000)$, tienden a emplear en mayor medida los super/hipermercados para la adquisición de alimentos en los que se incluye el plátano. Segovia-López, AlbornozGotera y Ortega-Soto (2007), reportaban que el nivel de ingresos está asociado de manera positiva con la elección del lugar de compra de alimentos frescos.

Las principales razones aducidas por los consumidores para la elección del lugar frecuente de compra son por cercanía al hogar $(57,3 \%)$ y por buenos precios (49,0\%) (ver figura 4). Otros aspectos como la frescura de los productos $(34,8 \%)$ y la calidad (32,3\%) poseen una menor importancia.

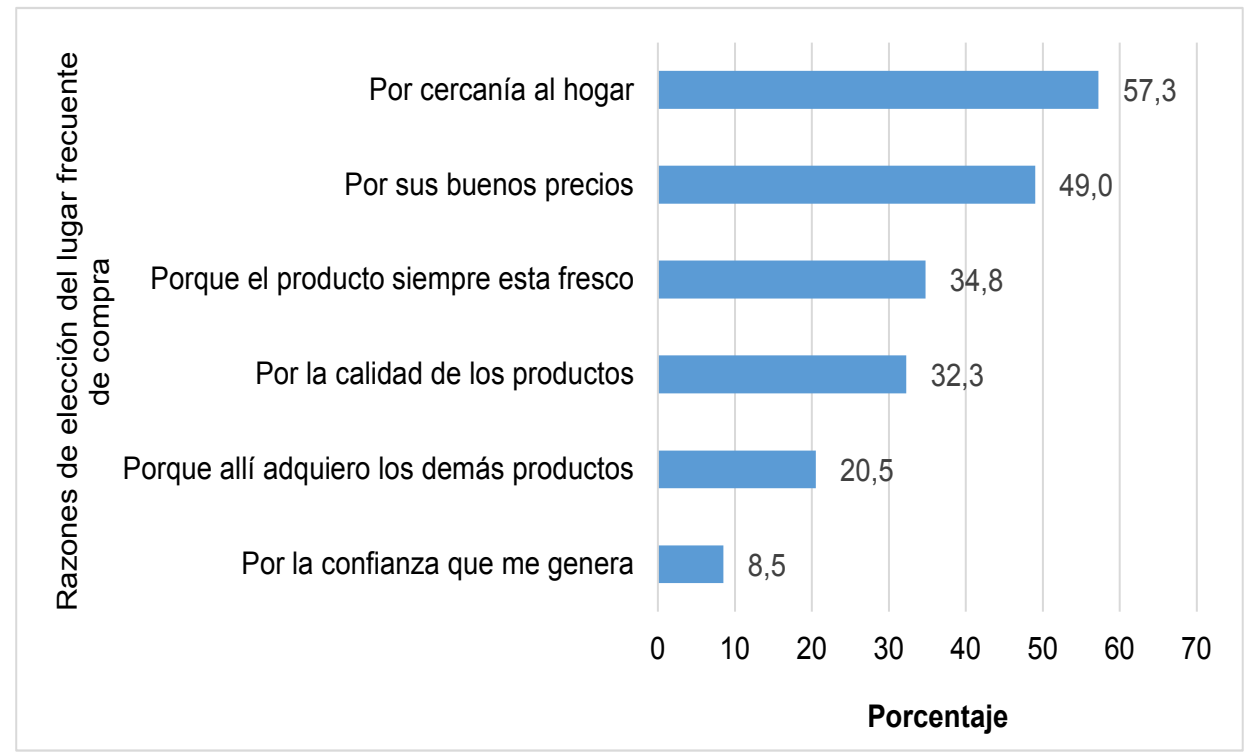

Figura 4. Razones de elección del lugar frecuente de compra

Las personas que suelen adquirir el plátano en los super/hipermercados, las tiendas tradicionales y micromercados, consideran en mayor medida la cercanía al hogar (test de Chi-cuadrado, $P=0,000$ ), por lo que podrían considerarse formatos de conveniencia. Sin embargo, los buenos precios está más asociado con las plazas de mercado (test de Chi-cuadrado, $P=0,000)$ mientras que una mejor calidad de los productos, está más asociado con los super/hipermercados (test de Chi- 
cuadrado, $P=0,000)$. Ávila, Herrera y Espinel (2009) reportaron, que en Ecuador los consumidores asocian las plazas de mercado con la consecución de mejores precios y las tiendas tradicionales con la cercanía al hogar y la adjudicación de créditos. Los mismos autores reportaron (Ávila, Herrera \& Espinel, 2009), que los supermercados están más asociados con la calidad de los productos y un mejor servicio. Por tanto, nuestros resultados son en cierta medida coincidentes con los descritos por otros autores.

En Ecuador al igual que en otros países del mundo, existe una gran diversidad de preparaciones culinarias a partir del plátano. De acuerdo con los encuestados, los patacones (61,5\%) y los bolones (52,3\%) son las principales preparaciones que se elaboran en los hogares ecuatorianos (ver figura 5). El patacón es una preparación culinaria que consiste en laminar trozos de la fruta para después ser sofreídos (PROECUADOR, 2015). Por su parte, el bolón es elaborado a partir de masa de plátano previamente cocinado en agua. En orden de importancia se encuentran las tortillas de verde (31,3\%), plátano asado (29,8\%), chifles (chips) $(24,3 \%)$ y sopas $(23,5 \%)$. Las demás preparaciones son menos efectuadas en los hogares de los encuestados. Honfo, Tenkouano y Coulibaly (2011), en Nigeria y Camerún, encontraron que las preparaciones a base de plátano más efectuadas eran el plátano cocido en agua, el plátano frito, los chips de plátano y el plátano asado. Preparaciones muy similares a las efectuadas en Ecuador. Existen en el mercado interno de Ecuador marcas comerciales de productos pre-cocinados que van en línea con los mayores usos dados al plátano en los hogares. Esto es un indicativo de la actividad innovadora que se viene generando alrededor de la cultura del consumo de plátano en Ecuador. En los patacones, se resalta la marca Patacón Chiflar. Por su parte, las marcas Facundo (de Guayaquil), Dulces y Empanadas Carmita (de Manta) y Joselo (de Quito), ofrecen empanadas a base de verde, mientras que la marca Delicias Express (de Quito) hace referencia a tortillas de verde. El chifle, es quizás el producto procesado a base del plátano más difundido en Ecuador. De este snack, existen decenas de marcas dirigidas a diversos segmentos del mercado. 


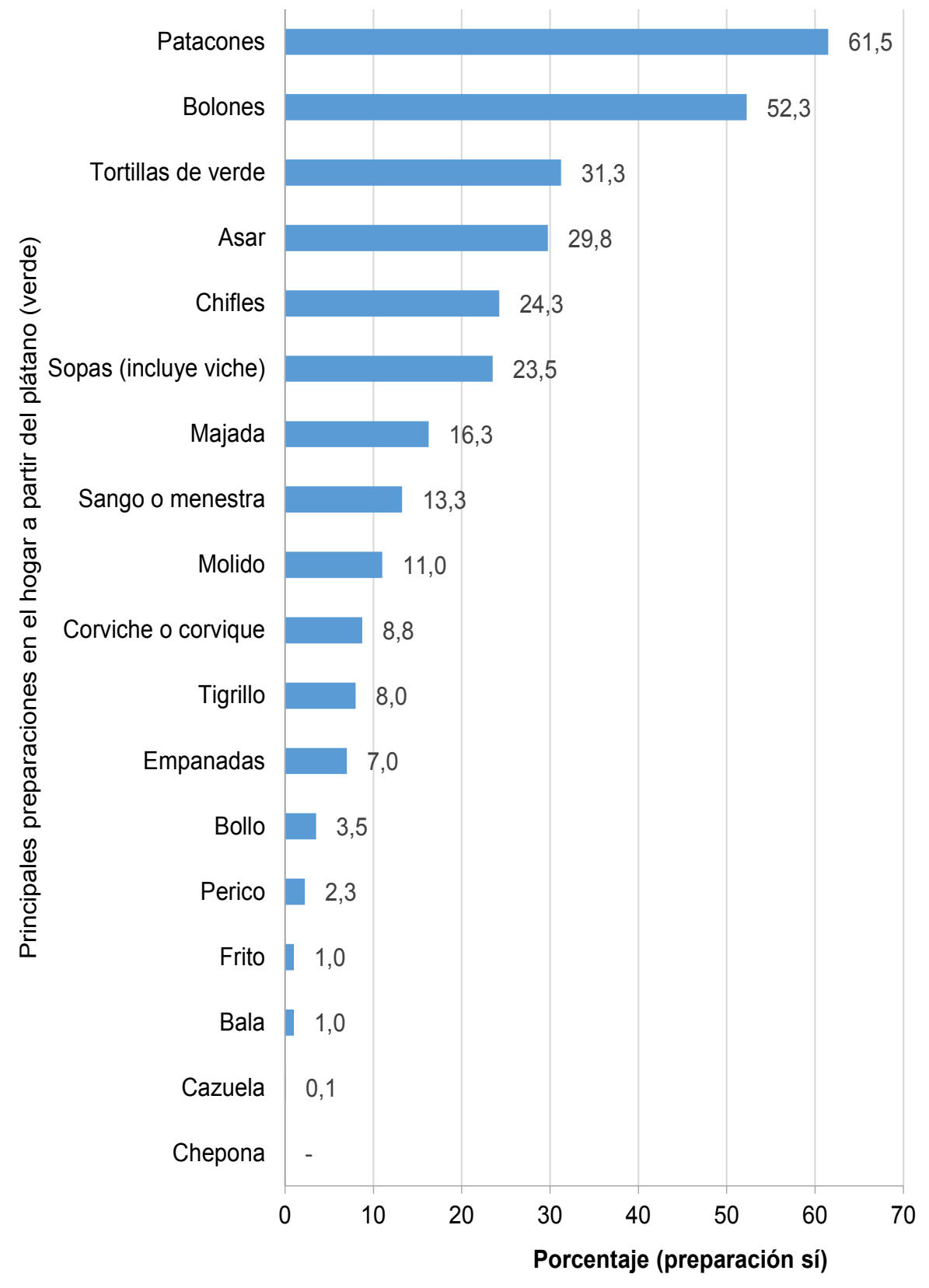

Figura 5. Principales preparaciones elaboradas en el hogar a partir del plátano (verde) 


\subsection{EXPERIMENTO DE ELECCIÓN}

En la tabla 3 se presentan los resultados del experimento de lección. Los signos de los efectos indican la dirección en que se afectan las utilidades de los consumidores a medida que la variable independiente asociada al efecto aumenta. Los resultados indican que si el plátano (verde) posee el sello de pequeños agricultores, aumenta la probabilidad de que este sea adquirido frente a uno que no lo posea, debido a que se afectan de manera positiva las utilidades de los consumidores. Por su parte, el uso de un sello de calidad tipo Premium Quality en el plátano frente a su no uso, también ejerce un efecto positivo sobre las utilidades generadas en los consumidores, lo que aumentaría la probabilidad de que el producto sea adquirido. Lo mismo sucede con el uso del sello de calidad de producción ecológica, en donde su uso en el plátano ejerce un efecto positivo sobre las utilidades de los consumidores frente a su no uso.

Tabla 3. Resultados del experimento de elección

\begin{tabular}{lllll}
\hline Atributo & Efecto & Sd Error & t -Ratio & DAP (USD \$) \\
\hline Producción ética & 0,074 & 0,018 & 4,219 & 0,020 \\
Calidad & 0,377 & 0,017 & 22,673 & 0,103 \\
Producción orgánica & 0,552 & 0,020 & 27,053 & 0,151 \\
& & & & \\
\hline Origen & & & & \\
\hline No específico & $-0,085$ & 0,039 & $-2,201$ & $-0,023$ \\
Manabí & $-0,205$ & 0,034 & $-5,942$ & $-0,056$ \\
Santo Domingo & 0,360 & 0,048 & 7,528 & 0,099 \\
Guayas & $-0,070$ & 0,033 & $-2,134$ & $-0,019$ \\
\hline Precio & & & & \\
\hline & $-3,646$ & 0,124 & $-29,356$ & \\
Constante & $-2,055$ & 0,055 & $-37,193$ & \\
\hline
\end{tabular}

Nota: $\mathrm{RLH}=0,50685$ 
Con relación al origen, el que el plátano sea originario de Santo Domingo frente a los demás empleados en el estudio, tiene un efecto positivo sobre las utilidades generadas en los consumidores, aumentado así la probabilidad de compra. Por su parte, los demás orígenes en contraposición con Santo Domingo, ejercen un efecto negativo sobre las utilidades de los consumidores, disminuyendo así la probabilidad de ser adquirido el producto. Dentro de estos efectos negativos, el más extremo es el origen Manabí, por lo que frente a las demás opciones generaría un efecto negativo sobre las utilidades de los consumidores. Si se tiene en cuenta que el mayor productor de plátano del Ecuador es la provincia de Manabí, y dentro de ésta el cantón El Carmen, esto hace suponer que podría haber cierta confusión en cuanto al "verdadero" origen del plátano en Ecuador, debido a que Santo Domingo es provincia limítrofe del cantón El Carmen.

En línea con la teoría económica, un aumento en el precio del producto lleva consigo un efecto negativo sobre las utilidades, lo que conduce a una reducción de la probabilidad de que el producto sea seleccionado. El valor negativo de la constante, indica que una persona obtiene mayor utilidad si selecciona la opción A o B frente a la posibilidad de no seleccionar ninguna de las dos. El valor correspondiente a los t ratio, indica que todos los parámetros estimados en el modelo son estadísticamente diferentes de cero y por tanto ejercen un efecto significativo sobre las utilidades de los consumidores.

Relativo a la disponibilidad a pagar (DAP), los resultados sugieren que la disponibilidad promedio máxima a pagar por el plátano con sello de pequeños productores es de USD $\$ 0,020$ por Kg más que el plátano producido sin sello de pequeños productores. Por su parte, los consumidores basados en la calidad del plátano estándar existente en el mercado, estarían dispuestos a pagar en promedio un máximo de USD $\$ 0,103$ más de sobreprecio por $\mathrm{Kg}$ de producto.

Por la existencia del sello de calidad orgánico en el plátano, los consumidores estarían dispuestos a pagar hasta un sobreprecio máximo promedio de USD \$0,151 por Kg del producto, frente a aquel que no posee dicho sello de calidad. En cuanto al origen, por el que éste provenga de Santo Domingo, los consumidores estarían dispuestos a pagar un sobreprecio máximo promedio de USD \$0,099 por Kg frente a otros orígenes. 
Dentro de los atributos ligados con la producción y que fueron incluidos en el experimento de elección, la producción orgánica es la que genera mayores utilidades y disponibilidades a pagar por parte de los consumidores. En orden de importancia le sigue el uso de un estándar de calidad voluntario que resalte la calidad del plátano tipo exportación. Dentro de los atributos de producción, el comercio justo es el que menos afecta las utilidades de los consumidores. Estos resultados van en cierta contraposición con la tendencia mundial en el norte global, en donde existe una mayor preferencia por el sello de comercio justo por encima de otros sellos de calidad como la producción orgánica (Doherty, Smith \& Parke, 2015). Esto podría deberse a las campañas del gobierno ecuatoriano por promover una alimentación sana y una producción amigable con el ambiente en todos los sectores de la economía.

\section{AGRADECIMIENTOS}

El manuscrito es resultado del proyecto de investigación "Análisis de Atributos de Calidad Superior Ligados con la Producción de Café Tostado y Molido, Cacao, Frutas Tropicales y Hortalizas Producidos por Pequeños Agricultores de la Provincia de Manabí financiado por el Proyecto Prometeo de la SENESCYT a Wilmer S. Sepúlveda. Los autores quieren agradecer a la Escuela de Economía de la Universidad Técnica de Manabí, por su apoyo en el desarrollo del proyecto.

\section{REFERENCIAS}

AFONSO, A. Caracterización de la dieta alimentaria de la población rural campesina de Manabí. Gobierno Provincial de Manabí, 2014.

ÁVILA, J.; HERRERA, P.; ESPINEL, R. Tendencias de los consumidores y mercados de productos agrícolas: un enfoque cualitativo. In: CONFERENCIA XLIV Asamblea Anual de CLADEA, Guayaquil-Ecuador, Noviembre 4-7, 2009.

BENALCAZAR, L.F. Plan estratégico para la entrega domiciliaria de productos orgánicos en un sector de la ciudad de Quito. (Tesis máster) - Universidad 
Internacional SEK, Facultad de Ciencias Económicas y Administrativas, Ecuador, Quito, 2009.

CAUTHEN, J.; JONES, D. Banana and Plantain Value Chain: West Africa. EPAR Brief No. 239. Evans School Policy Analysis and Research, 2013.

CORTÉS, M.; GAYOL, L. Cambio en las preferencias del consumidor de plátano en Puerto Rico, 2003-2008. The Journal of Agriculture of the University of Puerto Rico, v. 96, n. 12, p. 107-116, 2012.

DOHERTY, B.; SMITH, A.; PARKE, S. Fair Trade market creation and marketing in the Global South. Geoforum, Article in Press, 2015. Disponível em: <http://dx.doi. org/ 10.1016/j.geoforum.2015.04.015>.

DURY, S.; BRICAS, N.; TCHANGO-TCHANGO, J.; TEMPLE, L.; BIKOI, A. The determinants of urban plantain consumption in Cameroon. Food Quality and Preference, v. 13, p. 81-88, 2002.

ENSANUT - Encuesta Nacional de Salud y Nutrición. 2013. Ecuador 2011-2013. Instituto Nacional de Estadística y Censos, 2013.

FAOSTAT. Disponível em: < http://faostat.fao.org > Visitada en julio d 2015.

FORT, R.; RUBEN, R. The impact of fair trade on banana producers in northern Peru. En: RUBEN, Ruerd (Ed). The impact of fair trade, [s.l.; s.n.], 2008.

GAMBOA, E.M.; LÓPEZ, N.; PRADA, G.E.; FRANCO, J.T.; LANDÍNEZ, A. Factores asociados al consumo de frutas y verduras en Bucaramanga, Colombia. Archivos Latinoamericanos de Nutrición, v. 60, n. 3, p. 247-253, 2010.

GIRALDO TORO, A.; GIBERT, O.; RICCI, J.; DUFOUR, D.; MESTRES, C.; BOHUON, P. Digestibility prediction of cooked plantain flour as a function of water content and temperatura. Carbohydrate Polymers, v. 118, p. 257-265, 2015.

GRACIA, A.; LÓPEZ, B.; VIRUÉ, S. Disposición a pagar por zumos naturales: aplicación de un experimento de elección. ITEA, v. 107, n. 1, p. 21-32, 2011. 
HONFO, F.G.; TENKOUANO, A.; COULIBALY, O. Banana and plantain-based foods consumption by children and mothers in Cameroon and Southern Nigeria: A comparative study. African Journal of Food Science, v. 5, n. 5, p. 287-291, 2011.

INEC - Instituto Nacional de Estadísticas y Censos del Ecuador. Procesador de estadísticas agropecuarias del Ecuador -ESPAC. Disponível em: <http://www. ecuadorencifras.gob.ec/ procesador-de-estadisticas-agropecuarias-3/ $>$. Visitada en: julio de 2015.

JEKAYINFA, S.O.; OLA, F.A.; AFOLAYAN, S.O.; OGUNWALE, R. On-farm energy analysis of plantain production in Nigeria. Energy for Sustainable Development, v. 16, p. 339-343, 2012.

LYNAM, J.K. Market development and production potential for banana and plantain. Acta Horticulturae, v. 540, p. 39-53, 2000.

MCFADDEN, D. Decisiones económicas. Revista Asturiana de Economía -RAE, v. 21, p. 261-303, 2001.

Mendoza, C. Perspectivas de los agricultores y su incidencia en la producción agrícola diferenciada de calidad, de cacao, café, maracuyá y plátano, en la provincia de Manabí, durante el periodo 2012-2014. (Tesis de pregrado) - Escuela de Economía, Facultad de Ciencias Económicas y Administrativas, Universidad Técnica de Manabí, Ecuador: Portoviejo, 2015.

MOBERG, M. Fair trade and eastern Caribbean banana farmers: rhetoric and reality in the anti-globalization movement. Human Organization, v. 64, n. 1, 2005.

NOCEDA, C. et al. Field performance and (epi) genetic profile of plantain (Musa $\mathrm{AAB}$ ) clone 'CEMSA 3/4' plants micropropagated by temporary immersion systems. Scientia Horticulturae, v. 146, p. 65-75, 2012.

PARRADO, A.; MONTOYA, I.A. Características del consumo de vegetales en los estratos socioeconómicos medio y alto de Bogotá. Agronomía Colombiana, v. 25, n. 2, p. 377-382, 2007.

PROECUADOR. Análisis sectorial: plátano. Instituto de Promoción de Exportaciones 
e Inversiones, 2015.

SANTOS DE SOUZA, R. et al. Comportamento de compra dos consumidores de frutas, legumes e verduras na região central do Rio Grande do Sul. Ciência Rural, v. 38, n. 2, p. 511-517, 2007.

SEGOVIA-LÓPEZ, E.; ALBORNOZ-GOTERA, A.; ORTEGA-SOTO, L. Factores que determinan la selección del lugar de compra de carne de res en Maracaibo, Venezuela. Revista Cientifica (Maracaibo), v. 17, n. 4, 2007.

SEPÚLVEDA, W.S. Análisis de atributos de calidad superior ligados con la producción de café, cacao, maracuyá y plátano de la provincia de Manabí. Ecuador, Portoviejo: Universidad Técnica de Manabí, Gobierno Provincial de Manabí, 2015.

SEPULVEDA, W.S.; MAZA, M. T.; PARDOS, L. Aspects of quality related to the consumption and production of lamb meat. Consumers versus producers. Meat Science, v. 87, p. 366-372, 2011.

TCHANGO, J. et al. Plantain: Post-harvest Operations. Centre de Recherches Regionales sur Bananiers et Plantains, Cameroon (CRBP) - Food and Agriculture Organization of the United States (FAO), 1999.

TRADEMAP. Disponível em:<www.trademap.org > Visitada en: julio de 2015.

TRAUGER, A. Is bigger better? The small farm imaginary and fair trade banana production in the Dominican Republic. Annals of the Association of American Geographers, v. 104, n. 5, p. 1082-1100, 2015.

VERBEKE, W.; DEMEY, V.; BOSMANS, W.; VIAENE, J. Consumer versus producer expectations and motivations related to "superior" quality meat: qualitative research findings. Journal of Food Products Marketing, v. 11, n. 3, p. 27-41, 2005.

Recebido em: 12 de fevereiro 2016 Aceito em: 04 de outubro 2016 